\title{
Sensitivity Analysis of Heart Rate Variability Analysis Algorithms
}

\author{
Amanda Perez-Porro', María Palacios ${ }^{1}$, Gabriel Caffarena ${ }^{1}$, Constantino Antonio García ${ }^{1}$, Abraham \\ Otero ${ }^{1}$ \\ Department of Information Technology, Escuela Politécnica Superior, Universidad San Pablo-CEU, CEU Universities, \\ Urbanización Montepríncipe, 28660 Boadilla del Monte, Madrid, Spain \\ a.perezporro@usp.ceu.es, m.palacios14@usp.ceu.es, gabriel.caffarena@ceu.es, constantino.garciama@ceu.es, \\ Aotero@ceu.es
}

\section{Extended Abstract}

Heart rate variability analysis (HRV) is a non-invasive technique for the study of autonomic nervous system activity. HRV algorithms have a large number of configuration parameters, and it is not always clear how variations in some of these parameters influence the final result. This makes it difficult to choose appropriate parameter values [1], and hampers reproducibility of HRV studies [2].

We have carried out a sensitivity analysis of HRV algorithms to gain a better understanding of how changes in their parameters influence their results. For this end the R package RHRV was used; with about 500 monthly downloads this may be the most used HRV tool [3].

For the sensitivity analysis of the time domain algorithms and frequency domain algorithms (both based on the Shorttime Fourier transform and Wavelet transform), the MIT-BIH Normal Sinus Rhythm Database from Physionet was used. For the sensitivity analysis of ventricular beat filtering algorithms, the MIT-BIH Arrhythmia Database was used. The analysis was initially carried out using the parameters set by default in RHRV. Then, each parameter was systematically varied, reapplying the algorithms over the entire database for each variation of each parameter. The results obtained for each recording were stored in csv files for subsequent analysis.

Shapiro-Wilks test ruled out the normality of the results in all cases. Friedman nonparametric test was used to assess the significance of the differences in the results. When statistically significant differences were found, the specific parameter variations that presented significant differences were found with the Post-Hoc Conover test. This permits identifying from what amount of variation of a parameter the changes are significant.

Using this methodology, we have been able to identify the parameters for which even small variations produce statistically significant differences in the results of some HRV algorithm. For example, variations as small as $2 \%$ in the length of the time window used produced statistically significant differences in the time-domain index SDNN, although variations in the time window did not produce significant differences in the spectral power of the VLF, LF, and HF frequency bands.

\section{References}

[1] D. C. Jarrin, J. J. McGrath, S. Giovanniello, P. Poirier, and M. Lambert. (2012). Measurement fidelity of heart rate variability signal processing: The devil is in the details. International Journal of Psychophysiology, 86(1), 88-97.

[2] M. J. Lado, X. A. Vila, L. Rodríguez-Liñares, A. J. Méndez, D. N. Olivieri, and P. Félix. "Detecting sleep apnea by heart rate variability analysis: assessing the validity of databases and algorithms." Journal of medical systems 35, no. 4 (2011): 473-481.

[3] C. A. G. Martínez, A. Otero, X. A. Vila, M. J. L. Touriño, L. Rodríguez-Liñares, J, M. R. Presedo and A. J. M. Penín (2017). Heart rate variability analysis with the R package RHRV. Germany: Springer International Publishing.

\section{Funding}

This work was funded by the Ministry of Science, Innovation and Universities of Spain, grant RTI2018-095324-B-I00. 